\section{Sur le système d'équations intégrales à une infinité de fonctions inconnues}

par W. Pogorzelski (Warszawa)

I. Système du type de Fredholm

Dans ce travail nous étudions quelques systèmes d'équations intégrales à une infinité de fonctions inconnues. Les considérations qui suivent mettent en évidence la fécondité et la puissance de la méthode nouvelle d'étude des équations intégrales, fondée sur le théorème topologique de Schauder. Les problèmes que nous résolvons par cette méthode étaient irrésolubles à l'aide de l'analyse classique.

Rappelons tout d'abord les trois théorèmes suivants sur lesquels nous nous basons dans la suite.

THÉORÈME D'ARZELÀ. Si une famille de fonctions continues, définies dans une même région bornée, est uniformément bornée et les fonctions de cette famille sont équicontinues, alors on peut extraire de cette famille une suite de fonctions uniformément convergente.

THÉORÈME DE FRÉCHET ${ }^{2}$ ). Pour qu'un ensemble $X$ de points dans un espace métrique complet $E$ soit compact, il faut et il suffit que, pour tout $\varepsilon$ positif, il existe dans l'espace $E$ un ensemble compact $Y_{\varepsilon}$ tel qu'd chaque point $x$ de $X$ corresponde un point $y$ de $Y_{\varepsilon}$ dont la distance $\delta(x, y)$ du point $x$ serait inférieure à $\varepsilon$.

THÉOR亡̀ME DE SCHAUDER ${ }^{2}$ ). Si dans un espace complet, normé et linéaire, une operation continue transforme un ensemble de points borné, fermé et convexe, en un sous-ensemble compact de ce dernier, il existe au moins un point invariant de l'opération donnée.

Considérons maintenant un système d'équations intégrales, non linéaires et singulières, à une infinité dénombrable de fonctions inconnues

$$
\varphi_{1}(x), \varphi_{2}(x), \phi_{3}(x), \ldots, \varphi_{n}(x), \ldots
$$

1) M. Fréchet, Quelques propriétés des ensembles abstraits, Fumdamenta Mathematicae 12 (1928), p. 298-310.

2) J. Schauder, Der Fixpunktsatz in Funktionalräumen, Studia Mathematica 2 (1930), p. 171-180. du type suirant de Fredholm:

(2) $\quad \varphi_{n}(x)=\lambda \int_{\dot{\Omega}} \frac{F_{n}\left[x, y, \varphi_{1}(y), \varphi_{2}(y), \varphi_{3}(y), \ldots\right]}{|x y|^{\alpha_{n}}} d y \quad(n=1,2, \ldots, \infty)$

où $F_{n}\left(x, y, u_{1}, u_{2}, u_{3}, \ldots\right)$ sont des fonctions d'une infinité de variables définies dans la région fermée

(3) $\quad x \in \Omega, \quad y \in \Omega,\left|u_{1}\right| \leqslant R, \quad\left|u_{2}\right| \leqslant R, \ldots,\left|u_{n}\right| \leqslant R, \ldots$

On a désigné par $|x y|$ la distance des points $x$ et $y$ dans un domaine (fermé et borné) d'intégration $\Omega$ de l'espace euclidien à s dimensions; les exposants $a_{n}$ sont des constantes réelles données, non supérieures au nombre $\alpha<s$.

On suppose que les fonctions données $F_{n}\left(x, y, u_{1}, u_{2}, u_{3}, \ldots\right)$ soient continues en tout point de la région (3), e'est-à-dire que, pour tout point $\left(x_{0}, y_{0}, u_{1}^{0}, u_{2}^{0}, \ldots\right)$ de cette région et pour toute fonction $F_{n}$ séparément, on peut faire correspondre à chaque nombre positif $\varepsilon$ un nombre positif $\eta$ tel que

si

$$
\left|F_{n}\left(x_{0}, y_{0}, u_{1}^{0}, u_{2}^{0}, \ldots\right)-F_{n}\left(x, y, u_{1}, u_{2}, \ldots\right)\right|<\varepsilon
$$

$$
\left|x_{0} x\right|<\eta, \quad\left|y_{0} y\right|<\eta, \quad\left|u_{\nu}^{0}-u_{v}\right|<\eta \quad(\nu=1,2,3, \ldots) .
$$

En outre on admet que les fonctions $F_{n}$ sont uniformément bornées, c'est-à-dire qu'il existe un nombre positif $M$ tel que toutes les fonctions $F_{*}$ satisfont à l'inégalité

$$
\left|F_{n}\right| \leqslant M \text {. }
$$

Démontrons d'abord que chaque fonction $F_{n}$ séparément est uniformément continue dans la région (3). Considérons à cet effet un espace $\Pi$ composé de toutes les suites infinies $A\left(x, y, u_{1}, u_{2}, u_{3}, \ldots\right)$ où $x, y$ sont des points arbitraires du domaine $Q$, et $u_{1}, u_{2}, u_{3}, \ldots$ une suite réelle bornée, quelconque. Définissons la distance de deux points

(5) $\quad A\left(x, y, u_{1}, u_{2}, u_{3}, \ldots\right), \quad A^{\prime}\left(x^{\prime}, y^{\prime}, u_{1}^{\prime}, u_{2}^{\prime}, u_{3}^{\prime}, \ldots\right)$

de l'espace $\Pi$ par la formule

$$
\varrho\left(A, A^{\prime}\right)=\left|x x^{\prime}\right|+\left|y y^{\prime}\right|+\sum_{\nu=1}^{\infty} \varepsilon_{\nu}\left|u_{v}-u_{v}^{\prime}\right|,
$$

où $\sum \varepsilon_{v}$ est une série de nombres positifs convergente, arbitrairement choisie. L'ensemble (3) est alors borné dans l'espace $\Pi$; nous démontrerons qu'il est compact. En effet, pour $\varepsilon$ positif quelconque, considérons dans l'espace $\Pi$ l'ensemble de tous les points

$$
B_{\varepsilon}\left(x, y, u_{1}, u_{2}, u_{3}, \ldots, u_{N_{\varepsilon}}, 0,0,0, \ldots\right)
$$


dont les composantes à indice supérieur au nombre $N_{\varepsilon}$ tel que

$$
\sum_{\nu=N_{\varepsilon}}^{\infty} \varepsilon_{\nu}<\frac{\varepsilon}{2 R}
$$

sont nulles. A chaque point $A\left(x, y, u_{1}, u_{2}, u_{3}, \ldots\right)$ de l'ensemble (3), on peut done faire correspondre un point $B_{\varepsilon}$ tel que

$$
\varrho\left(A, B_{\varepsilon}\right)<\varepsilon .
$$

Mais l'ensemble des points $B_{\varepsilon}$ dans l'espace $\Pi$ est évidemment compact par conséquent, d'après le théorème cité de Fréchet, l'ensemble (3) est aussi compact dans l'espace $\Pi$. Il en résulte que toute fonction $F_{n}$ sépa rément est uniformément continue dans l'ensemble (3), c'est-à-dire que l'on a, pour deux points quelconques (5) de (3), l'inégalité

$$
\left|F_{n}(A)-F_{n}\left(A^{\prime}\right)\right|<\varepsilon
$$

si $\varrho\left(A, A^{\prime}\right)<\eta$, ou bien si $\left|x x^{\prime}\right|<\eta_{1},\left|y y^{\prime}\right|<\eta_{1},\left|u_{\nu}-u_{\nu}^{\prime}\right|<\eta_{1}$, où

$$
\eta_{1}=\frac{\eta}{2+\sum_{\nu} \varepsilon_{\nu}}
$$

( $\eta$ ne dépend que de $\varepsilon$ et $n$ ).

Pour l'étude du système (2), considérons l'espace fonctionnel $E^{\infty}$ de toutes les suites infinies

$$
\left[\varphi_{1}(x), \varphi_{2}(x), \ldots, \varphi_{n}(x), \ldots\right]
$$

bornées, composées des fonctions déterminées et continues dans le domaine fermé $\Omega$. Définissons la distance des deux points $U\left\{\varphi_{1}\right\}$ et $V\left\{g_{n}\right\}$ de l'espace $E^{\infty}$ par la formule

$$
\delta(U, V)=\sum_{n=1}^{\infty} \varepsilon_{n} \sup \left|\varphi_{n}-g_{n}\right|,
$$

où $\sum \varepsilon_{n}$ est une série numérique aux termes positifs convergente, arbitrairement choisie. La norme du point $U\left\{\varphi_{n}\right\}$ sera définie par sa distance de l'origine $\Theta(0,0,0, \ldots)$ :

$$
\|U\|=\delta(U, \Theta)=\sum_{n=1}^{\infty} \varepsilon_{n} \sup \left|\varphi_{n}\right| .
$$

La définition (9) vérifie toutes les conditions admises pour l'espace métrique.

II est évident que la condition de Cauchy est suffisante pour la convergence d'une suite arbitraire de points $U_{1}, U_{2}, U_{3}, \ldots$ de l'espace $E^{\infty}$ et, par conséquent, cet espace est complet. En outre l'espace $E^{\infty}$ sera $l i$ néaire, si l'on admet les définitions suivantes du produit d'un point $V\left\{\varphi_{n}\right\}$ par le nombre réel $\gamma$ et de la somme des deux points $U\left\{\varphi_{n}\right\}$ et $\nabla\left\{g_{n}\right\}$ :

$$
\gamma U=\left\{\gamma \varphi_{n}\right\}, \quad U+\nabla=\left\{\varphi_{n}+g_{n}\right\} .
$$

Soit maintenant, dans l'espace $E^{\infty}$, un ensemble fermé $S$ de tous les points $U\left\{\varphi_{n}\right\}$ vérifiant l'inégalité

$$
\left|\varphi_{n}\right| \leqslant R .
$$

Cet ensemble est convexe, puisque tous les points du segment rectiligne

$$
(1-\gamma) U+\gamma \nabla \text {, }
$$

joignant les points $U\left\{\varphi_{n}\right\}$ et $V\left\{g_{n}\right\}$ de $S$, vérifient l'inégalité

$$
\left|(1-\gamma) \varphi_{n}+\gamma g_{n}\right| \leqslant(1-\gamma)\left|\varphi_{n}\right|+\gamma\left|g_{n}\right| \leqslant R
$$

quand $\gamma$ varie dans l'intervalle $(0,1)$; donc ces points appartiennent à l'ensemble $S$.

D'après la forme de l'équation intégrale proposée (2), transformons maintenant l'ensemble $S$ en faisant correspondre à tout point $U\left\{\varphi_{n}\right\}$ de cet ensemble un point $U^{\prime}\left\{\psi_{n}\right\}$ de $E^{\infty}$ dont les composantes sont déterminées par les formules

(13) $\quad \psi_{n}(x)=\lambda \int_{\Omega} \frac{F_{n}\left[x, y, \varphi_{1}(y), \varphi_{2}(y), \varphi_{3}(y), \ldots\right]}{|x y|^{\alpha_{n}}} d y \quad(n=1,2, \ldots, \infty)$.

Le point $U^{\prime}\left\{\psi_{n}\right\}$ appartient à l'ensemble $S$, si le paramètre $\lambda$ est suffisamment petit pour qu'on ait

$$
|\lambda| M \int_{\Omega} \frac{d y}{|x y|^{a_{n}}} \leqslant R
$$

où $I I$ désigne la borne supérieure de l'ensemble des fonctions $\left|F_{n}\right|$. Or on a

$$
\int_{\Omega} \frac{d y}{|x y|^{\alpha_{n}}} \leqslant \int_{0}^{L} \frac{\omega_{s} \varrho^{s-1}}{\varrho^{a_{n}}} d \varrho=\frac{\omega_{s}}{s-a_{n}} L^{s-\alpha_{n}} \leqslant \frac{\omega_{s}}{s-\alpha} L^{\prime}=l,
$$

où $L$ est le diamètre du domaine $\Omega, \omega_{s}$ - l'aire de la surface sphérique à $s$ dimensions

$$
\omega_{s}=\frac{2(l \cdot \bar{\pi})^{s}}{\Gamma(s / 2)}
$$

en outre on a $L^{\prime}=L^{s}$ si $L>1$, et $L^{\prime}=L$ si $L \leqslant 1$. La condition (9) sera done satisfaite si

$$
|\lambda| \leqslant \frac{R}{M \pi}
$$


et l'ensemble $S^{\prime}$ des points transformés de $S$ fera partie de cet ensemble. Pour prouver, d'après le théorème de Schauder, l'existence du point invariant de la transformation (13) il faut montrer encore que la transformation fonctionnelle (13) est continue et que l'ensemble transformé $S^{\prime}$ est compact.

Soit donc dans l'ensemble $S$ une suite infinie de points $\left\{U_{\nu}\left(\varphi_{1}^{\nu}, \varphi_{2}^{\prime}, \ldots\right)\right\}$ convergente vers le point $U\left(\varphi_{1}, \varphi_{2}, \ldots\right)$. On a alors

$$
\delta\left(U_{p}, U\right)=\sum_{n=1}^{\infty} \varepsilon_{n} \sup \left|\varphi_{n}^{\nu}-\varphi_{n}\right| \rightarrow 0, \quad \text { si } \quad \nu \rightarrow \infty
$$

Si $\left\{U_{v}^{\prime}\left(\psi_{1}^{\nu}, \psi_{2}^{\nu}, \ldots\right)\right\}$ est la suite de points qui correspondent aux points $U_{v}$ dans la transformation $(13)$ et $U^{\prime}\left(\psi_{1}, \psi_{2}, \ldots\right)$ correspond au point limite $U$, on a alors pour toute valeur entière positive de $n$ et $\nu$

$\psi_{n}^{v}(x)-\psi_{n}(x)=\lambda \int_{\Omega} \frac{F_{n}\left[x, y, \varphi_{1}^{v}(y), \varphi_{2}^{v}(y), \ldots\right]-F_{n}\left[x, y, \varphi_{1}(y), \varphi_{2}(y), \ldots\right]}{|x y|^{a_{n}}} d y$.

D'après la supposition (17), toutes les différences $\varphi_{n}^{\nu}-\varphi_{n}$ tendent uniformément vers zéro dans le domaine $\Omega$ si $\nu \rightarrow \infty$; en outre les fonctions $F_{n}$ sont uniformément continues séparément, on peut donc faire correspondre à tout $\varepsilon$ positif l'indice $N_{n}(\varepsilon)$ pour qu'on ait

$$
\left|F_{n}\left[x, y, \varphi_{1}^{y}(y), \varphi_{2}^{y}(y), \ldots\right]-F_{n}\left[x, y, \varphi_{1}(y), \varphi_{2}(y), \ldots\right]\right|<\varepsilon
$$

si $\nu>N_{n}(\varepsilon)$, où $N_{n}(\varepsilon)$ peut dépendre de $n$. On aura ainsi

$$
\left|\psi_{n}^{\nu}(x)-\psi_{n}(x)\right| \leqslant|\lambda| \varepsilon \int_{\Omega} \frac{d y}{|x y|^{a_{n}}} \leqslant|\lambda| \varepsilon l
$$

si $\nu>N_{n}(\varepsilon)$. Remarquons maintenant que les fonctions $\psi_{n}^{\prime \prime}$ vérifient, quels que soient $n$ et $\nu$, l'inégalité

$$
\left|\psi_{n}^{\nu}(x)\right| \leqslant R .
$$

On peut done choisir un indice $n_{0}(\varepsilon)$ qui ne dépend que de $\varepsilon$ et qui soit suffisamment grand pour qu'on ait

$$
\sum_{n=n_{0}(\varepsilon)+1}^{\infty} \varepsilon_{n} \sup \left|\psi_{n}^{\nu}-\psi_{n}\right| \leqslant \varepsilon
$$

quel que soit $\nu$. L'indice $n_{0}(\varepsilon)$ étant fixé, on aura alors, d'après (19), l'inégalité

$$
\sum_{n=1}^{n_{0}(\varepsilon)} \varepsilon_{n} \sup \left|\psi_{n}^{\nu}-\psi_{n}\right| \leqslant|\lambda| \varepsilon l A
$$

(où $A=\sum_{n=0}^{\infty} \varepsilon_{n}$ ) pour $y>\bar{N}(\varepsilon)$, où $\bar{N}(\varepsilon) \geqslant N_{n}(\varepsilon)$ si $n=1,2, \ldots, n_{0}(\varepsilon)$. Il en résulte, d'après les inégalités (20) et (21), que

$$
\delta\left(U_{v}^{\prime},{U^{\prime}}^{\prime}\right)=\sum_{n=1}^{\infty} \varepsilon_{n} \sup \left|\psi_{n}^{v}-\psi_{n}\right| \leqslant(1+|\lambda| l A) \varepsilon
$$

si $\nu>\bar{N}(\varepsilon)$; donc $\delta\left(U_{v}^{\prime}, U^{\prime}\right) \rightarrow 0$ si $\nu \rightarrow \infty$, et la transformation fonctionnelle, définie par les relations (13), est continue dans l'ensemble $\$$

II reste à démontrer que l'ensemble transformé $S^{\prime}$ est compact. A cet effet démontrons que les composantes (13) de tous les points transformés, correspondant à l'indice $n$ fixé, sont équicontinues, c'est-à-dire qu'à tout $\varepsilon$ positif correspond un nombre $\eta$ tel que toutes les fonctions (8), pour $n$ fixé, vérifient l'inégalité

$$
\left|\psi_{u}(x)-\psi_{n}\left(x^{\prime}\right)\right|<\varepsilon, \quad \text { si } \quad\left|x x^{\prime}\right|<\eta
$$

Décomposons donc la différence (22) en deux parties

$$
\begin{aligned}
\psi_{n}(x)-\psi_{n}\left(x^{\prime}\right)=\lambda \int_{\Omega} \frac{F_{n}\left[x, y, \varphi_{1}(y), \varphi_{2}(y), \ldots\right]-F_{n}\left[x^{\prime}, y, \varphi_{1}(y), \varphi_{2}(y), \ldots\right]}{|x y|^{\alpha_{n}}} d y+ \\
\quad+\lambda \int_{i} F_{n}\left[x^{\prime}, y, \varphi_{1}(y), \varphi_{2}(y), \ldots\right]\left(\frac{1}{|x y|^{a_{n}}}-\frac{1}{\left|x^{\prime} y\right|^{a_{n}}}\right) d y .
\end{aligned}
$$

D'après la continuité des fonctions $F_{n}$, toute fonction $F_{n}$ vérifie l'inégalité

$$
\text { (24) } \quad|\lambda|\left|F_{n}\left[x, y, \varphi_{1}(y), \varphi_{2}(y), \ldots\right]-F_{n}\left[x, y, \varphi_{1}(y), \varphi_{2}(y), \ldots\right]\right|<\frac{\varepsilon}{2 l}
$$

si $\left|x x^{\prime}\right|<\eta_{1}$, où le nombre positif $\eta_{1}$ dépend du nombre positif arbitraire $\varepsilon$. $\Pi$ en résulte, d'après l'inégalité (15), que le module de la première intégrale (23) sera inférieur à $\varepsilon / 2$ si $\left|x x^{\prime}\right|<\eta_{1}$. Pour rendre la seconde intégrale (23) inférieure à $\varepsilon / 2$ en module par rapprochement des points $x$ et $x^{\prime}$, remarquons que l'on a

$$
\begin{gathered}
\lambda \int_{\Omega} F_{n}\left[x^{\prime}, y, \varphi_{1}(y), \varphi_{2}^{\circ}(y), \ldots\right]\left(\frac{1}{|x y|^{\alpha_{n}}}-\frac{1}{\left|x^{\prime} y\right|^{\alpha_{n}}}\right) d y \mid \\
\leqslant M|\lambda| \int_{\Omega} \mid \frac{1}{|x y|^{\alpha_{n}}}-\frac{1}{\left|x^{\prime} y\right|^{\alpha_{n}}} d y .
\end{gathered}
$$


Soit maintenant une sphère $K$ de centre $x$ située à l'intérieur du domaine $\Omega$, et décomposons l'intégrale à droite dans l'inégalité (25) en deux parties

(26)

$$
\int_{\Omega}\left|\frac{1}{|x y|^{\alpha_{n}}}-\frac{1}{\left|x^{\prime} y\right|^{a_{n}}}\right| d y=\int_{K}\left|\frac{1}{|x y|^{\alpha_{n}}}-\frac{1}{\left|\dot{x}^{\prime} y\right|^{\alpha_{n}}}\right| d y+\int_{\Omega-K}\left|\frac{1}{|x y|^{\alpha_{n}}}-\frac{1}{\left|x^{\prime} y\right|^{\alpha_{n}}}\right| d y
$$

étendues à la sphère $K$ et à la partie extérieure $\Omega-K$. On pent choisir le rayon $r_{\varepsilon}$ de la sphère $K$ suffisamment petit pour qu'on ait

$$
\int_{K} \frac{d y}{\left|x^{\prime} y\right|^{\alpha}}<\frac{\varepsilon}{6 M|\lambda|}
$$

en tout point $x^{\prime}$ et pour tous les exposants $\alpha_{n} \leqslant \alpha \leqslant s$. Le point $x$ étant extérieur au domaine $\Omega-K$ et le rayon $r_{\varepsilon}$ étant fixé, on peut faire correspondre maintenant au nombre $\varepsilon$ un nombre $\eta_{2}$ indépendant de $x$ et de $n$ tel qu'on ait

$$
\left|\frac{1}{|x y|^{\alpha_{n}}}-\frac{1}{\left|x^{\prime} y\right|^{\alpha_{n}}}\right|<\frac{\varepsilon}{6 M V|\lambda|}
$$

si $\left|x x^{\prime}\right|<\eta_{2}$ et où $y$ est un point arbitraire à l'extérieur de la sphère $K$; $V$ désigne le volume du domaine $\Omega$. Finalement, d'après les formules (25), (26), (27), (28), on conclue que le module de l'intégrale (25) sera inférieur à $\varepsilon / 2$ si $\left|x x^{\prime}\right|<\eta_{2}$; on aura donc

$$
\left|\psi_{n}(x)-\psi_{n}\left(x^{\prime}\right)\right|<\varepsilon
$$

pour toute fonction $\psi_{n}$ et pour tout couple de points $x$ et $x^{\prime}$ du domaine $\Omega$, dont la distance est inférieure aux nombres $\eta_{1}, \eta_{2}$. Par conséquent, les fonctions $\psi_{n}$ sont équicontinues pour $n$ fixé.

A chaque point $U^{\prime}\left(\psi_{1}, \psi_{2}, \ldots, \infty\right)$ de l'ensemble transformé $S^{\prime}$, faisons correspondre un point $U_{\varepsilon}^{\prime \prime}\left(\psi_{1}, \psi_{2}, \ldots, \psi_{N_{\varepsilon}}, 0,0,0, \ldots\right)$ dont les composantes à indice supérieur au nombre $N_{\varepsilon}$ s'annullent, tel que l'on ait

$$
\delta\left(U^{\prime}, U_{\varepsilon}^{\prime \prime}\right)=\sum_{n=N_{\varepsilon}+1}^{\infty} \varepsilon_{n} \sup \left|\psi_{n}\right|<\varepsilon
$$

où $\varepsilon$ est un nombre positif arbitrairement choisi. Or l'ensemble de tous les points ${V_{\varepsilon}^{\prime \prime}}_{\varepsilon}$, dont les composantes au nombre finie de $N_{\varepsilon}$ sont différentes de zéro, est compact d'après la propriété démontrée (22) et le théorème d'Arzelà. Il en résulte, d'aprês le théorème de Fréchet cité au début, que l'ensemble transformé $S^{\prime}$ est compaict. Toutes les conditions du théorème de Schauder sont donc satisfaites et l'existence dans l'ensemble $S$ du point $\left(\varphi_{1}, \varphi_{2}, \ldots, \infty\right)$ invariant par rapport à la transformation (8), c'est-à-dire l'existence d'une solution du système d'équations intégrales (2) à une infinité de fonctions inconnues se trouve prouvée.
II. Système d'équations du type elliptique

Soit maintenant le système d'équations différentielles

$$
\Delta U_{n}=\lambda F_{n}\left(A, u_{1}, u_{2}, u_{3}, \ldots\right) \quad(n=1,2,3, \ldots, \infty)
$$

à une infinité de fonctions inconnues $u_{1}(A), u_{2}(A), u_{3}(A), \ldots$ dans un domaine borné $\Omega$ de l'espace euclidien à trois dimensions; $\Delta$ désigne l'opérateur de Laplace.

On recherche le système de fonctions $\left\{u_{n}(A)\right\}$ qui satisfont aux équations (30) à l'intérieur du domaine $\Omega$ et qui s'annullent aux points $P$ de la surface fermée $S$ limitant le domaine $\Omega$

$$
u_{n}(A) \rightarrow 0, \quad \text { si } A \rightarrow P \in S .
$$

On suppose que les fonctions $F_{n}\left(A, u_{1}, u_{2}, u_{3}, \ldots\right)$ d'une infinité de variables sont déterminées et uniformément bornées dans le domaine fermé

$$
A \in \Omega, \quad\left|u_{n}\right| \leqslant R \quad(n=1,2,3, \ldots, \infty),
$$

et qu'elles y vérifient la condition d'Hölder, c'est-à-dire l'inégalité

$$
\begin{gathered}
\left|F_{n}\left(A, u_{1}, u_{2}, u_{3}, \ldots\right)-F_{n}\left(\bar{A}, \bar{u}_{1}, \bar{u}_{2}, \bar{u}_{3}, \ldots\right)\right| \\
<k_{n}|A \bar{A}|^{\mu}+\sum_{p=1}^{\infty}\left|k_{n}^{\nu}\right| u_{p}-\left.\bar{u}_{p}\right|^{\mu} \quad(0<\mu \leqslant 1) .
\end{gathered}
$$

On suppose en outre que la série numérique des coëfficients d'Hölder

$$
x_{n}=\sum_{n=1}^{\infty} k_{n}^{y}
$$

est convergente quel que soit $n$.

Pour trouver une solution du problème posé, considérons le système d'équations intégrales suivantes:

$$
u_{n}(A)=-\frac{\lambda}{4 \pi} \int_{\Omega} G(A, B) F_{n}\left[B, u_{1}(B), u_{2}(B), \ldots\right] d \tau_{B}
$$

à une infinité de fonctions inconnues $u_{1}(A), u_{2}(A), u_{3}(A), \ldots G(A, B)$ est la fonction de Green habituelle relative à la surface $\$$. On a donc, pour deux points intérieurs $A, B$,

$$
0<G(A, B)=\frac{1}{|A B|}-g(A, B)<\frac{1}{|A B|},
$$

$g(A, B)$ étant harmonique même si $A$ coïncide avec $B$. Le système (34) est donc semblable au système (2) étudié précédemment. Les fonctions $F_{n}$ sont uniformément continues, d'après la supposition (33). En outre on démontre dans la théorie du potentiel, que l'estimation de 
la continuité des intégrales de la forme (34) n'exige que la connaissance de la limite supérieure des fonctions $\left|F_{n}\right|$, donc ces intégrales forment une famille de fonctions équicontinues. Par conséqent, en repétant le raisonnement de la première partie, on démontre l'existence d'une solution du système $(34)$ composée des fonctions continues $u_{1}(A), u_{2}(A)$, $u_{3}(A), \ldots$, si la valeur absolue du paramètre $\lambda$. vérifie l'inégalité (16), qui, dans le cas actuel, est la suivante:

$$
|\lambda| \leqslant \frac{2 R}{M L^{2}}
$$

où $M$ désigne la borne supérieure de l'ensemble des fonctions $\left|F_{n}\right|$, et $L$ - le diamètre du domaine $\Omega$.

D'après la propriété de la fonction de Green, les fonctions $u_{n}(A)$ satisfont à la condition limite (31). Il reste à démontrer qu'elles vérifient les équations différentielles (30). Or, si les fonctions continues $u_{n}(A)$ satisfont aux équations (34), elles admettent les dérivées $u_{n}^{\prime}(A)$ par rapport aux coordonnées du point $A$ sous la forme

$$
u_{n}^{\prime}(A)=-\frac{\lambda}{4 \pi} \int_{\Omega} G_{A}^{\prime}(A, B) F_{n}\left[B, u_{1}(B), u_{2}(B), \ldots\right] d \tau_{B} .
$$

On sait bien que les dérivées de la fonction de Green vérifient l'inégalité

$$
\left|G_{A}^{\prime}(A, B)\right|<\frac{c}{|A B|^{2}}
$$

( $c$ étant une constante) pour tous les couples $A, B$ dans $\Omega$. Donc, les dérivées des fonctions $u_{n}(A)$ sont bornées dans le domaine $\Omega$, notamment on a

$$
\left|u_{n}^{\prime}(A)\right|<\frac{|\lambda| M c}{4 \pi} \int_{\Omega} \frac{d \tau_{B}}{|A B|^{2}} \leqslant|\lambda| M c L .
$$

Il en résulte que les fonctions $u_{n}(A)$ satisfont à l'inégalité

$$
\left|u_{n}(A)-u_{n}(\bar{A})\right|<3|\lambda| M c L|A \bar{A}|,
$$

et, par conséquent, d'après la propriété (33), les fonctions composées $F_{n}\left[B, u_{1}(B), u_{2}(B), \ldots\right]$ vérifient l'inégalité

$$
\begin{gathered}
\left|F_{n}\left[B, u_{1}(B), u_{2}(B), \ldots\right]-F_{n}\left[\bar{B}, u_{1}(\bar{B}), u_{2}(\bar{B}), \ldots\right]\right| \\
<k_{n}|B \bar{B}|^{\mu}+\sum_{\nu=1}^{\infty} k_{n}^{\nu}\left|u_{\nu}(B)-u_{\nu}(\bar{B})\right|^{\mu} \leqslant\left(k_{n}+|3 \lambda M c L|^{\mu} \chi_{n}\right)|B \bar{B}|^{\mu},
\end{gathered}
$$

donc elles vérifient la condition d'Hölder. On en tire, d'après le théorème bien connu de la théorie du potentiel, que les fonctions $u_{n}(A)$, formant la solution du système d'équations intégrales (34), admettent les dérivées secondes en tout point intérieur $A$ du domaine $\Omega$, et vérifient le système d'équations différéntielles (30) ainsi que la condition au bord $S$ du domaine $\Omega$.

\section{Système du type de Volterra}

On étudiera encore le système d'équations singulières à une infinité de fonctions inconnues $\varphi_{1}, \varphi_{2}, \ldots$ de la forme

(41) $\int_{0}^{x} \frac{K_{n}(x, y)}{(x-y)^{\alpha_{n}}} \varphi_{n}(y) d y+\lambda \int_{0}^{x} \frac{F_{n}\left[x, y, \varphi_{1}(y), \varphi_{2}(y), \ldots\right]}{(x-y)^{\alpha_{n}}} d y=f_{n}(x)$,

où les constantes $\alpha_{n}$ sont inférieures à l'unité. On suppose que les fonctions $K_{n}(x, y), f_{n}(x)$ sont déterminées et continues dans la région fermée

$$
0 \leqslant x \leqslant a, \quad 0 \leqslant y \leqslant a,
$$

et qu'on a $f_{n}(0)=0$.

Les fonctions d'une infinité de variables $F_{n}\left[x, y, u_{1}, u_{2}, u_{3}, \ldots\right]$ sont déterminées et continues dans la région

$$
0 \leqslant x \leqslant a, \quad 0 \leqslant y \leqslant a, \quad\left|u_{v}\right| \leqslant R, \quad \nu=1,2,3, \ldots
$$

En outre on suppose que les fonctions $K_{n}(x, y), f_{n}(x), F_{n}\left(x, y, u_{1}, u_{2}, \ldots\right)$ admettent les dérivées par rapport à la variable $x$ dans les régions (42) et (43) et que ces dérivées sont continues. On peut transformer le système (41) en système d'équations

$$
\begin{aligned}
\int_{0}^{z} \frac{d x}{(z-x)^{1-\alpha_{n}}}\left[\int_{0}^{x} \frac{K_{n}(x, y)}{(x-y)^{a_{n}}} \varphi_{n}(y) d y+\right. \\
\left.\quad+\lambda \int_{0}^{x} \frac{F_{n}\left[x, y, \varphi_{1}(y), \varphi_{2}(y), \ldots\right]}{(x-y)^{\alpha_{n}}} d y-f_{n}(x)\right]=0 .
\end{aligned}
$$

Il en résulte, par l'application de la transformation de Dirichlet, que le système (41) est équivalent au système d'équations régulières (45) $\quad \int_{0}^{z} K_{n}^{*}(z, y) \varphi_{n}(y) d y+\lambda \int_{0}^{z} F_{n}^{*}\left[z, y, \varphi_{1}(y) \varphi_{2}(y), \ldots\right] d y=f_{n}^{*}(z)$ $n=1,2, \ldots, \infty)$ où l'on a posé

$$
\begin{aligned}
K_{n}^{*}(z, y) & =\int_{y}^{z} \frac{K_{n}(x, y)}{(z-x)^{1-a_{n}}(x-y)^{a_{n}}} d x, \\
F_{n}^{*}\left(z, y, u_{1}, u_{2}, \ldots\right) & =\int_{y}^{z} \frac{F_{n}\left(x, y, u_{1}, u_{2}, \ldots\right)}{(z-x)^{1-a_{n}}(x-y)^{a_{n}}} d x, \\
f_{n}^{*}(z) & =\int_{0}^{z} \frac{f_{n}(x)}{(z-x)^{1-a_{n}}} d x .
\end{aligned}
$$


D'après les propriétés admises pour les fonctions $K_{n}, F_{n}, f_{n}$, les fonctions (46) sont déterminées, bornées, continues et admettent les dérivées par rapport à la variable $z$, continues dans les régions (42) et (43). Désignons ces dérivées par $K_{n}^{* \prime}(x, y), f_{n}^{* \prime}(z), F_{n}^{* \prime}\left(z, y, u_{1}, u_{2}, u_{3}, \ldots\right)$. En dérivant les denx côtés des équations (45) par rapport à la variable $z$, et en remarquant que ces côtés s'annullent pour $z=0$, on peut affirmer que le système (41) est équivalent au système

$$
\begin{aligned}
K_{n}^{*}(z, z) \varphi_{n}(z)+\int_{0}^{z} K_{n}^{* \prime}(z, y) \varphi_{n}(y) d y+\lambda F_{n}^{*}\left[z, z, \varphi_{1}(z), \varphi_{2}(z), \ldots\right] & + \\
& +\lambda \int_{0}^{z} F_{n}^{* \prime}\left[z, y, \varphi_{1}(y), \varphi_{2}(y), \ldots\right] d y=f_{n}^{* \prime}(z) .
\end{aligned}
$$

Il est facile de vérifier que

$$
K_{n}^{*}(z, z)=K_{n}(z, z) \frac{\pi}{\sin \alpha_{n} \pi} .
$$

En admettant que $K_{n}(z, z) \neq 0$, et en appliquant maintenant la solution bien connue de Volterra, on peut affirmer enfin que le système donné (41) est équivalent au système d'équations intégrales

$$
\begin{aligned}
\varphi_{n}(z)= & -\lambda \int_{0}^{z} \Phi_{n}\left[z, y, \varphi_{1}(y), \varphi_{2}(y), \ldots\right] d y- \\
& -\lambda F_{n}^{* *}\left[z, \varphi_{1}(z), \varphi_{2}(z), . . . \cdot\right]+f_{n}^{* *}(z),
\end{aligned}
$$

où les fonctions $\Phi_{n}, F_{n}^{* *}, f_{n}^{* *}$ sont déterminées par les formules

$(49)$

$$
\begin{aligned}
\Phi\left(z, y, u_{1}, u_{2}, \ldots\right)= & \int_{y}^{z} \frac{F_{n}^{* \prime}\left(\bar{y}, y, u_{1}, u_{2}, \ldots\right)}{K_{n}^{*}(\bar{y}, \bar{y})} \Re_{n}(z, y) d y+ \\
& +\frac{F_{n}^{*}\left(y, y, u_{1}, u_{2}, \ldots\right)}{K_{n}^{*}(y, y)} \Re_{n}(z, y)+\frac{F_{n}^{* \prime}\left(z, y, u_{1}, u_{2}, \ldots\right)}{K_{n}^{*}(z, z)},
\end{aligned}
$$

$$
\begin{aligned}
F_{n}^{* *}\left(z, u_{1}, u_{2}, \ldots\right) & =\frac{F_{n}^{*}\left(z, z, u_{1}, u_{2}, \ldots\right)}{K_{n}^{*}(z, z)} \\
f_{n}^{* *}(z) & =\frac{f_{n}^{* \prime}(z)}{K_{n}^{*}(z, z)}+\int_{0}^{z} \vartheta_{n}(z, y) \frac{f_{n}^{* \prime}(y)}{K_{n}^{*}(y, y)} d y
\end{aligned}
$$

où $\mathfrak{N}_{n}(z, y)$ désigne le noyau résolvant de

$$
N_{n}(z, y)=-\frac{K_{n}^{* \prime}(z, y)}{K_{n}^{*}(z, z)}
$$

c'est-à-dire c'est la somme de la série

$$
\mathfrak{N}_{n}(z, y)=N_{n}(z, y)+\sum_{p=1}^{\infty} N_{n}^{p}(z, y)
$$

où

$$
N_{n}^{\nu}(z, y)=\int_{y}^{z} N_{n}(z, \bar{y}) N_{n}^{\nu-1}(\bar{y}, y) d \bar{y} \quad\left(N_{n}^{0}=N_{n}\right)
$$

Toutes les fonctions (49) sont bornées, d'après la supposition $f_{n}(0)=0$, en outre, elles sont continues dans l'ensemble fermé (43). En admettant que l'ensemble des bornes supérieures des modules des fonctions (49), pour $n=1,2, \ldots, \infty$, est borné, on peut répéter le raisonnement de la première partie. On considère donc dans l'espace $E^{\infty}$, composé des suites $\left\{\varphi_{n}(x)\right\}$ de fonctions continues, une transformation fonctionnelle

$$
\begin{aligned}
\psi_{n}(z)= & -\lambda \int_{0}^{z} \Phi_{n}\left[z, y, \varphi_{1}(y), \varphi_{2}(y), \ldots\right] d y- \\
& -\lambda F_{n}^{* *}\left[z, \varphi_{1}(z), \varphi_{2}(z), \ldots\right]+f_{n}^{* *}(z)
\end{aligned}
$$

et on constate, d'après le théorème de Schauder, l'existence d'une solution $\left\{\varphi_{n}(x)\right\}$ du système d'équations intégrales (41), le module de $\lambda$ et de la variable $z$ étant suffisamment petit.

\section{INSTYTUT MATEMATYCZNY POLSKIEJ AKADEMII NAUK}

INSTITUT MATHF́MATIQUE DE L'ACADEMIE POLONAISE DES SCIENCES 